\title{
POTENCIALIDADES EDUCATIVAS DE LAS WEBRADIOS UNIVERSITARIAS. UNA APROXIMACIÓN DESDE LA PERSPECTIVA DE DOCENTES Y DISCENTES
}

\author{
Piñeiro- Otero, Teresa ${ }^{1}$ \\ Universidade da Coruña \\ Teresa.pineiro@udc.es \\ Ramos, Fernando ${ }^{2}$ \\ Universidade de Aveiro. \\ fernando.ramos@ua.pt
}

Material original autorizado para su primera publicación en la revista académica REDMARKA. Revista Digital de Marketing Aplicado.

https://doi.org/10.17979/redma.2011.01.06.4722

\section{RESUMEN}

La evolución de la radiofonía hertziana a la web supuso una renovación del producto sonoro que, con la incorporación de otro tipo de elementos inherentes al sistema digital, alcanza una proyección más allá del ámbito estrictamente sonoro.

Las características del medio radiofónico en Internet ofrecen múltiples

\footnotetext{
${ }^{1}$ Doctora en Comunicación por la Universidad de Vigo, Master en Docencia e Investigación educativa para la Educación Superior por la Universidad Española de Educación a Distancia y profesora en la Facultad de Ciencias de la Comunicación de la Universidade da Coruña. Su ámbito de estudio más reciente se ha centrado en el fenómeno de Internet como plataforma de desarrollo de una radio universitaria, ámbito en el que profundizó durante una estancia de investigación en el polo de Aveiro del CETAC.MEDIA- Centro de Estudos das Tecnologias e Ciências da Comunicação.

${ }^{2}$ Profesor Catedrático del Departamento de Comunicação e Arte da Universidade de Aveiro (Portugal). Coordinador Científico del CETAC.MEDIA-Centro de Estudos das Tecnologias e Ciências da Comunicação. Director del programa doctoral en Informação e Comunicação em Plataformas Digitais, programa conjunto de las Universidades de Aveiro y de Porto. Sus intereses de investigación se centran en el estudio de la relación entre las tecnologías de la comunicación y los nuevos contextos de enseñanza-aprendizaje en la Educación Superior.
} 
potencialidades para las radios universitarias que, en el medio digital, están registrando un crecimiento exponencial debido a que presentan nuevas formas de creación, emisión, difusión e intercambio de información.

El presente trabajo tiene por objeto profundizar en las potencialidades que la web brinda a las radios universitarias desde una perspectiva educativa, así como conocer las percepciones que estudiantes y profesores -como sus principales públicos- tienen sobre este ámbito.

Una aproximación -parte de un trabajo exploratorio en torno a la creación de una webradio en la Universidade de Aveiro- para la que se seleccionaron sendas muestras de conveniencia, de docentes y discentes, que llevó a adoptar para cada una de ellas una metodología y una herramienta de investigación diferentes.

Para el estudio de las percepciones de los alumnos respecto a las capacidades educativas de las webradios universitarias se optó por un cuestionario, mientras que en el caso de los profesores dicho trabajo fue abordado a través de entrevistas personales.

Los datos recabados por ambas herramientas subrayaron la escasa importancia de la vertiente educativa en la concepción que estudiantes y profesores presentan sobre los contenidos de una radio universitaria. Una concepción que podría interferir en el aprovechamiento de las potencialidades que la webradio presenta para el proceso de enseñanza-aprendizaje.

Palabras clave. Webradio, radios universitarias, educación

\section{ABSTRACT}

EDUCATION POTENTIAL OF UNIVERSITY WEBRADIO. AN APPROACH FROM THE PERSPECTIVE OF TEACHERS AND SPEAKERS.

Changes from analogical radio to webradio has involved an audible product 
updating that, with digital media elements incorporation, achieves a projection further of the audible field.

Internet radio characteristics offers numerous potentials for university radios. Radio stations are living an exponential growth on the web and it pushes up new forms of creation, broadcast and diffusion.

This paper aims to deepen in the possibilities that the web offers to college radio from an educational perspective and understanding the perceptions that students and teachers as a major public have in this field.

Key words Webradio, university radios, education.

\section{RESUMO}

A evoluação da radiofonía hertziana para ambiente digital implicou uma renovação do produto sonoro que, com a incorporação de outros elementos inerentes ao sistema digital, alcança uma projeção além do âmbito sonoro.

As características do media radiofónico em Internet, da webrádio, oferece múltiplas potencialidades para as rádios universitárias, que estão a registar um crescimento exponencial dado a web proporcionar a emergência de novas formas de criação, emissão, difusão e partilha.

O presente trabalho tem por objeto aprofundar nas potencialidades que a web brinda às rádios universitárias desde uma perspetiva educativa, quer como conhecer as percepções que estudantes e professores -como principais públicos- têm em este âmbito.

Palavras-chave. Webrádio, rádios universitárias, educação. 


\section{Introducción}

La incorporación de la radio al ámbito de la universidad, así como el aprovechamiento de sus potencialidades educativas por la comunidad académica, no constituye un fenómeno reciente. La primera iniciativa de radio en el contexto universitario, llevada a cabo en la Universidad de Wisconsin, data de 1919 (Faus, 1973); antes incluso que las primeras licencias de radiodifusión.

Pese a su pronta aparición, la implantación de las radios universitarias fue bastante inferior, por ejemplo, al de las múltiples cabeceras desarrolladas dentro de la comunidad académica (Fidalgo Díez, 2009). Una situación que se ha invertido con la incorporación de las nuevas tecnologías de la información y de la comunicación al proceso de emisión-transmisión y su proyección a través de Internet.

En este sentido, el traspaso de la radio hertziana a la webradio, la denominada "radiomorfosis" (Prata, 2008), supuso la renovación del producto radiofónico con la incorporación de componentes inherentes al sistema digital. En Internet la radio implementa sus estrategias comunicativas más allá del ámbito estrictamente sonoro (González Conde, 2010, p. 55), desarrolla un nuevo concepto de programación más próximo a la personalización de contenidos (Albarran y Pitts, 2001, p. 172), pierde fugacidad y favorece la creación y cohesión de comunidades de oyentes más segmentadas (Prietsman, 2002, p. 226); unos oyentes que en la web cuentan con nuevas formas de participación basadas en la interactividad (Priestman, 2002, p. 228; Tolson, 2006; Nyre y AlaFossi, 2008, p. 44).

La esencia del medio radiofónico en Internet ofrece múltiples potencialidades para las radios universitarias. Unas emisoras que están viviendo un importante desarrollo en la web debido a las facilidades de carácter tecnológico y legal que esta forma de radiodifusión ofrece, y que han propiciado el surgimiento de nuevos modos de creación, emisión y difusión (Teixeira, Perona Páez y Gonçalves, 2010, p. 184). De hecho, desde las primeras iniciativas de web 
radios universitarias, que tuvieron lugar hacia finales de la década de los 90 , este fenómeno no hizo más que expandirse.

Siguiendo a Kunh (2001, p. 7-8) el medio radiofónico en Internet no sólo ha ganado nuevas potencialidades sino que ha permitido "rescatar algumas utopias adormecidas, como o rádio interativo, o rádioalternativo, o rádio educador"; utopías de algún modo presentes en la radiofonía universitaria.

Tomando como referencia la perspectiva educativa, el objeto del presente trabajo ha sido el de profundizar en las potencialidades que la web brinda a las radios universitarias como plataformas de una comunidad educativa, así como conocer las percepciones que sobre este ámbito presentan sus principales públicos del ecosistema académico: alumnos y profesores.

\section{Radios Universitarias}

Por radio universitaria se entiende un tipo de emisora con características propias de las radios comunitarias y de las radios educativas, que opera dentro de una comunidad académica. Unas emisoras que pueden constituir, siguiendo a Sauls (1995, p.1), un proyecto global de carácter institucional que implica a toda la comunidad universitaria, o una iniciativa desarrollada por una entidad de ámbito más restringido (facultad, asociación de estudiantes, profesor-alumnos de una determinada materia, etc.).

El fenómeno de la radiofonía universitaria ha ido evolucionando desde las primeras estaciones experimentales y hoy en día presenta múltiples configuraciones en función de su soporte tecnológico de difusión (FM, AM, web), su alcance (desde un circuito cerrado de un centro concreto a una comunidad de oyentes más amplia), sus objetivos (educación, divulgación, entretenimiento) o modelos de gestión (Sauls, 1995, p. 2 y ss.).

Para González Conde (2001, p. 476-477) entre los rasgos característicos de las radios universitarias se encuentran su vocación de servicio público, su 
especialización en divulgación científico-cultural, su facilidad de acceso a los miembros de la comunidad, el ofrecer un enfoque orientado hacia un público mayoritariamente joven o el constituir un soporte para la publicación de contenidos de carácter docente. Estas características configuran, para las radios universitarias, una programación y contenidos alternativos (Caton en Sauls, 1998), con frecuencia diametralmente opuestos a los vehiculados por las emisoras comerciales (Waits, 2008, p. 83; Perona Páez, 2009; p. 109).

"From its beginnings college radio had has 'rebel heritage', a reputation for 'being differente', for 'bucking trends and bucking tradition'” (McLung, Mims y Hong, 2003, p. 167). Circunstancia que ha propiciado que determinados autores como Desztich y McClung (2007, p. 210) se refieran a las radios universitarias como clubs de élite fundamentados en una cultura de independencia, diferencia y exclusividad (Desztich y McClung, 2007, p. 210).

Perona Páez ha señalado como elemento distintivo de las radios universitarias una programación de tipo mosaico donde tienen cabida cualquier tipo de géneros y de temas (Perona Páez, 2009, p. 109). "More specifically, the alternative programming is primarily made up of three types: entertainment, information, and instruction" (Caton, 1979 en Sauls, 1998).

A pesar de las posibilidades de diversificación de su oferta programática, la "Contemporary Hit Radio" continúa siendo la fórmula más explotada por las emisoras universitarias (Perona Páez, 2009, p. 109). Una fórmula que supuso el foco de atención de la mayor parte de estudios sobre contenidos de las emisoras académicas (Rothenbuhler, 1987; Sauls, 1998 y 2000; Wall, 2007; Mcclung y Desztich, 2007; Waits, 2008; Baker, 2010; entre otros).

No obstante, el mayor peso de la programación musical no debe eclipsar el potencial educativo de las radios universitarias. Teixeira, Perona Páez y Daher coinciden con Merayo, Perona Páez y Veloso en considerar las radios universitarias como una categoría dentro de las emisoras de vertiente cultural y educativa, al igual que las radios comunitarias, populares, formativas autárquicas y escolares. Una tipología de emisoras que tienen en común contar 
con unos objetivos no comerciales y su vocación social (Merayo, Perona Páez y Veloso en Teixeira, Perona Páez y Daher, 2010, p. 179).

Las emisoras universitarias presentan, además, una función cohesionadora que sumada al hecho de operar dentro de un ecosistema académico, les confiere de determinadas características propias de las emisoras comunitarias como la proximidad de su público objetivo, la desvinculación de un padrón comercial estático o la posibilidad de producción de contenidos por los propios miembros de la comunidad (Caldeira y Simeone, 2006, p. 67). Independientemente del tipo de configuración que presenten, el principal objetivo de toda radio universitaria es el de ofrecer un servicio a la comunidad; sea ésta una comunidad estrictamente académica u otra más amplia (Sauls, 1995, p. 2).

Esta concepción de la radio universitaria sufrió una importante transformación a finales de la década de los 90 con la llegada de la radiofonía web, a la que hay que sumar la crisis de algunos de los elementos implícitos en la estructura y modelos de gestión de dichas emisoras.

\section{Webradios universitarias y educación 2.0}

Las potencialidades que ofrece Internet para la radiodifusión, en especial desde la perspectiva de la interactividad, favorecieron la aparición de numerosas plataformas on-line con orientación educacional, entre las que se incluyen las radios universitarias (Perona Páez, 2009, p. 107).

Las webradios han enriquecido la oferta de las radios hertzianas con espacios multimedia complementarios y otro tipo de funcionalidades (foros, blogs, chats, etc.) que además de facilitar la interacción emisor-receptor anulan el carácter secuencial, la fugacidad y la verticalidad propias de la difusión analógica (Perona Páez, 2009, p. 108).

Estas nuevas potencialidades de la radio en Internet favorecen su utilización 
dentro de una comunidad universitaria con una multiplicidad de objetivos. Desde una perspectiva educacional propiamente dicha, las radios web favorecen la creación y consolidación de comunidades virtuales de aprendizaje como plataformas de divulgación educativa y cultural (Teixeira e Da Silva, 2009, p. 5).

Por otra parte, la incorporación de la webradio al ámbito de la radiodifusión universitaria favorece la conexión con las nuevas generaciones de estudiantes; los "nativos digitales". Unos estudiantes que se configuran como los principales oyentes/usuarios de la radio en Internet, así como de los diferentes servicios que vehicula (Rose y Lenski, 2007; o Baker, 2010).

\subsection{Dos vertientes de la radio universitaria: experimentación y educación.}

En Radio Dadá Manifiesto Corbett (1993, p. 83) se refirió al hecho de que:

Within the academic institution, the radio station has become a place of experimentation and teaching. The tension between these two functions... is belied by the 'educational role' of college radio. Is it a place where one learns how to do radio-cum-industry, or is it a space outside of that circuit, where the industry itself can be questioned, pushed, or perhaps ignored?

Estas dos vertientes, experimental y educacional, aparecen abordadas de forma sucinta en los estudios sobre radiofonía web en el ámbito universitario. Así, pese a que el desarrollo de las radios universitarias en Internet dio lugar a numerosas investigaciones, se puede considerar un cierto sesgo en el tratamiento de innovación y educación en las radios universitarias online.

Por un lado, la experimentación de las radios universitarias con las potencialidades del medio digital recibió un abordaje, fundamentalmente, del tipo de estudios de caso (Wallace, 2008; Pessoa, 2008; Perona Páez, 2009; Teixeira et al., 2008; Teixeira y Da Silva, 2009a; Costa Filho, 2009; Teixeira et al., 2010 ; Baker, 2010; Machado Velho, s/d; entre otros). 
Por otro, a pesar de que las emisoras universitarias constituyen una de las tipologías más conocidas de la radio educativa, esta vertiente quedó relegada en el corpus teórico en torno a la radiofonía universitaria en detrimento de otros aspectos. La atención de la mayor parte de los trabajos realizados se centra en las nuevas posibilidades de distribución de contenidos sonoros, la interactividad y el hipermedia desde la perspectiva del entretenimiento, especialmente de la programación de tipo musical alternativa (de particular relevancia en el ámbito de los Estados Unidos: Wall, 2007; Mcclung y Desztich, 2007; Waits, 2008; Baker, 2010, entre otros).

Entre los estudios que tratan las potencialidades educativas de las webradios universitarias destacan aquellos que se centran en nuevas formas de distribución de contenidos de audio, como los podcast (Diegues y Coutinho, 2009), o en los edu-sites (Perona Páez, 2009; Barbeito, 2010); dos elementos que pese a formar parte del universo de la radiofonía web no se pueden considerar radios educativas per se.

\subsection{Radios universitarias 2.0. Potencialidades educativas.}

Las adecuación de las características de la radiofonía web al contexto de la radio universitaria ha puesto de relevancia una serie de potencialidades para la educación. Potencialidades entre las que destacan el refuerzo de la conexión con los nativos digitales, mayor entidad de los contenidos educativos, perfecta adecuación a un contexto multitasking, el paso hacia un paradigma de formación always on \& everywhere, la interactividad, la configuración de espacios virtuales de aprendizaje o el desarrollo de una formación profesional.

\subsubsection{Refuerzo de la conexión con los nativos digitales}

El uso de las webradios universitarias con finalidad educativa permite establecer más de un canal de interacción entre docentes y discentes; unos discentes que pertenecen a los "nativos digitales" (Prensky, 2001).

Las características de este grupo pueden considerarse en convergencia con una serie de posibilidades que brinda la radiofonía web: "Nomadismo, 
individualismo, customização e personalização, exibição e voyerismo, espaço público e privado, memória da geração sob demanda e um perfil jovem em transformação" (Rodrigues da Cunha, 2010, p. 182). Convergencia que no debe ser desaprovechada por las radios universitarias.

\subsubsection{Mayor entidad de los contenidos educativos}

En las webradios universitarias los contenidos de carácter educativo pueden enriquecerse con la incorporación de nuevos elementos de carácter multimedia, susceptibles de mejorar la experiencia de aprendizaje.

Además de posibilitar diferentes niveles de profundidad en el consumo de los contenidos, el hiperaudio (o hipermedia) facilita al oyente/usuario seguir una ruta de contenidos en función de sus intereses; ruta que puede delimitar públicos concretos dentro de la comunidad universitaria.

\subsubsection{Perfecta adecuación a un contexto multitasking}

La adecuación a un contexto multitasking se configura como una de las principales ventajas para su consumo. En efecto, una de las características de los nativos digitales es su tendencia a la realización de diversas tareas en simultáneo o -en el ámbito estrictamente mediático- a la exposición simultánea a diversos medios (Cardoso, Espanha y Araujo, 2009, p. 3).

Las potencialidades del consumo de contenidos educativos en las webradios universitarias se pueden multiplicar siempre que su mensaje sonoro, desvinculado de los elementos de carácter multimedia, esté dotado de sentido completo.

\subsubsection{Always on \& everywhere}

La exponencial implantación de los iPod, reproductores MP3 y demás dispositivos móviles entre los estudiantes universitarios favorece la escucha de la radio web y, en particular, de uno de sus elementos distintivos: los podcast. En efecto, siguiendo a Weintraub (en McClung, Pompper y Kinally, 2007, p. 
116) la portabilidad del medio radiofónico contribuyó a su incorporación al estilo de vida de los jóvenes.

El desarrollo de la telefonía móvil, cuya concepción mudó del terminal telefónico a la pantalla, y la penetración alcanzada por los dispositivos portátiles multimedia (MP3, PDA, PSP...) favorecieron la aparición del paradigma del always on. "Handheld devices ... are well suited to accommodate new types of audience participation. The devices are intimately associated with flesh-and-blood individuals, and are typically off-limits to most other persons" (Nyre y Ala-Fossi, 2008, p. 43).

Esta "cuarta pantalla" (Aguado-Terrón y Martínez-Martínez, 2009) ha flexibilizado el consumo de las webradios universitarias que, una vez liberado de cualquier frontera espacial (gracias a la movilidad de los receptores) y temporal (con la inclusión de los contenidos on demand que permiten su revisión) incrementa las ocasiones de uso everywhere.

\subsubsection{Interactividad}

Las posibilidades de interactividad que presenta la webradio adquiere especial interés en el ámbito de una comunidad universitaria, debido su poder de cohesión social, expresión de valores y formación de su identidad como grupo (Kischinhesky, 2009, p.234). Una interactividad que hizo surgir la figura del prosumidor (Islas-Carmona, 2008), un oyente, a la vez, consumidor y productor de contenidos en la web.

La interacción de estos oyentes en las diversas redes sociales establece una relación de comunicación entre ellos y con el conductor; relación que permite establecer un feedback en tiempo real respecto de los contenidos vehiculados. Así permite la consolidación de medios colaborativos basados en una sola red social o que conjugan diversas redes y herramientas de la web 2.0 (wiki, galerías virtuales de fotografías, plataformas audiovisuales, agregadores de noticias, etc.)

\subsubsection{Espacios virtuales de aprendizaje.}


La perspectiva de la webradio universitaria como una emisora que difunde contenidos a través de una plataforma en la web permite considerar diversas posibilidades para la creación de espacios virtuales de aprendizaje en función del grado de integración de funcionalidades orientadas a dar soporte a las actividades de enseñanza-aprendizaje en el site radiofónico. Esta integración puede limitarse a la incorporación en la web de un enlace en el campus virtual de la universidad 0 , al contrario, poner a disposición un espacio dentro de la web que dé soporte a múltiples actividades de interacción docentes-discentes o entre estudiantes.

Este espacio virtual se puede convertir en un lugar de intercambio de material relacionado con una determinada unidad curricular, curso o titulación. Esos contenidos puede ir desde apuntes, lecturas de apoyo, ejercicios, grabaciones de aulas (lecturecasting) o indicaciones prácticas, foros de debate, información sobre eventos académicos, etc. (Teixeira, 2009). El acceso e intercambio de materiales en estas plataformas de webradio universitaria favorecen la relación entre colegas de clase o de curso, formando comunidades virtuales que, con frecuencia, son utilizadas para establecer relaciones personales, circunstancia que puede ser un estímulo para el aprendizaje y desarrollo de prácticas pedagógicas (Pessoa, s/d).

\subsubsection{Formación profesional}

Algunos de los estudios desarrollados en el ámbito de la radio universitaria analizan la importancia de estas emisoras para los estudiantes como una práctica y/o trampolín para el desarrollo de su carrera profesional en el ámbito radiofónico (Sauls, 1995; Pesha, 1997; Desztich y McLung, 2007). De facto, una gran parte de las radios universitarias se crean al abrigo de las escuelas de comunicación con la intención de crear un contexto para el desarrollo de prácticas lo más próximo posible a la realidad.

Sin embargo, la webradio universitaria ofrece posibilidades de "simulación profesional" más allá de las prácticas de comunicación. Se puede perfilar, por ejemplo, el desarrollo de un debate político en el ámbito de las clases de 
Ciencias Políticas o una unidad didáctica para la educación a distancia, para los estudiantes de Educación.

En el caso concreto de la Universidade de Aveiro entre cuya oferta formativa se encuentra la titulación en Música, resultaría interesante incluir emisiones en directo de conciertos de los alumnos. Práctica que, además de ofrecer oportunidades performativas y de familiarizar a los estudiantes con los diferentes sistemas de captación y digitalización del sonido, permitiría dar a conocer los artistas entre la comunidad universitaria.

\section{Metodología}

En busca de conocer la viabilidad, desde una perspectiva educativa, de la puesta en marcha de una plataforma de webradio en la Universidade de Aveiro (en adelante UA) se consideró interesante desarrollar un estudio de las percepciones que estudiantes y profesores tienen de las potencialidades didácticas de una plataforma de estas características.

Un trabajo en el que se realizó una aproximación a los dos públicos principales de una webradio universitaria, adoptando para cada uno de ellos una muestra, metodología y herramienta de estudio diferentes.

\subsection{Estudiantes}

Los estudiantes se configuran, siguiendo a Rose y Lenski (2007) y Baker (2010), como los destinatarios por antonomasia de una webradio universitaria $y$, por lo tanto, de sus contenidos educativos. Circunstancia que se revela de especial interés a la hora de conocer sus percepciones en torno a las posibilidades didácticas de la webradio.

Para ello se tomó como muestra de análisis un total de 55 sujetos, pertenecientes a dos grupos diferentes alumnos de la UA: estudiantes de posgrado en comunicación (máster y doctorado) y estudiantes-investigadores 
de nacionalidad extranjera, de diversas áreas científicas.

Gráfico 1. Muestra de estudiantes en función de su nacionalidad.

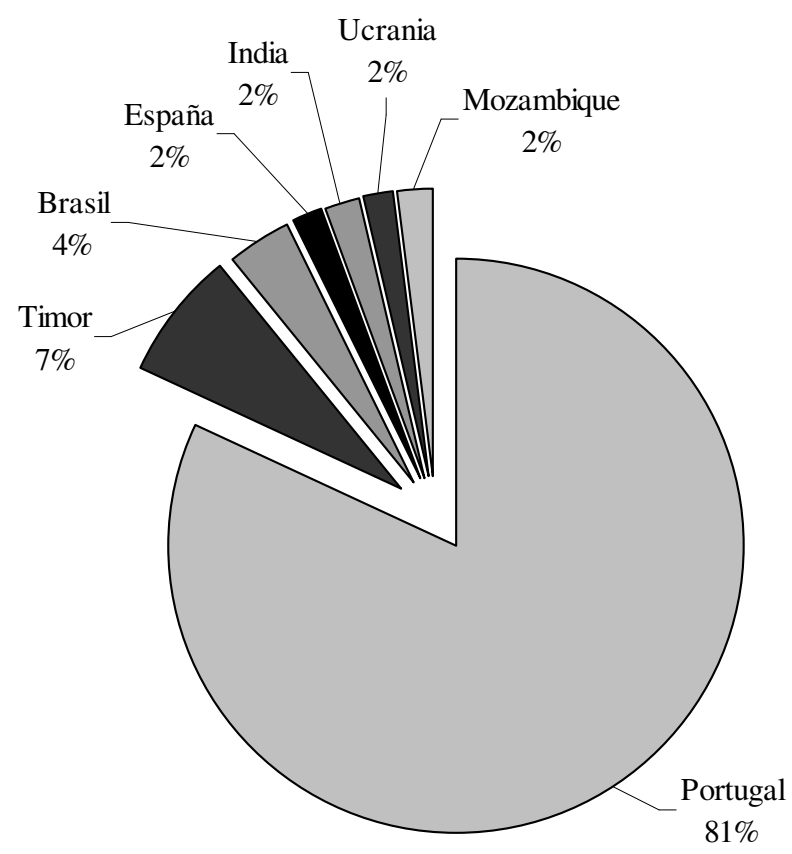

Fuente. Elaboración propia.

La selección de ambas muestras de conveniencia se realizó en función de criterios diferentes.

El primer grupo, de 45 estudiantes de posgrado en comunicación, fue seleccionado atendiendo a la presunción de que, debido a su especialidad, presentarían un consumo activo de los medios de comunicación así como una perspectiva interesante sobre contenidos y programación que podría ser incorporada en la siguiente fase de la investigación (con una muestra mayor y estadísticamente representativa de la comunidad universitaria de Aveiro). 
La elección del segundo grupo, de 10 estudiantes de posgrado extranjeros (todos ellos usuarios de la residencia de posgrados de la propia universidad), se debe a la importancia que los estudiantes-investigadores extranjeros adquieren en la comunidad universitaria de Aveiro. Esta relevancia propició que se considerase la inclusión de contenidos didácticos destinados a cubrir algunas de las necesidades de este grupo concreto como, por ejemplo, un programa de aprendizaje de portugués.

Para conocer las percepciones que dicha muestra presenta en torno a las posibilidades educativas de las webradios se optó por el cuestionario como herramienta de trabajo. Una herramienta con la que se recabaron datos de carácter cuantitativo y cualitativo a través del establecimiento de diferentes tipologías de preguntas (según el tipo de respuesta: abierta, cerrada, múltiple, sí o no, escala de Likert o jeraquizada).

El cuestionario final centró su atención en aquellas preferencias y percepciones de los alumnos en relación a las webradios universitarias en general, tomando como referencia el estudio de Free (2005) que identifica como motivaciones de adhesión a una plataforma de radio web la conveniencia (horario, facilidad de acceso), la calidad de contenidos (con la posibilidad de enriquecer el audio con contenidos multiplataforma) o la diversificación de las opciones de programación, adaptada a las máximas de informar, formar y entretener que siguiendo a Catón (en Sauls, 1998) deben estar presentes en toda radio universitaria. Unas máximas entre las que se destacó la formativa, en cuanto se corresponde con el objeto de estudio del presente trabajo.

Con la intención de validar este cuestionario se tomó como referencia un grupo de control de cinco individuos (pertenecientes a la población objeto de estudio), que permitió mejorar la formulación de algunas preguntas así como la coherencia global y organización de esta herramienta de recogida de datos.

\subsection{Profesores}

Los docentes constituyen otro de los principales públicos de las radios 
universitarias, cobrando especial protagonismo desde una perspectiva educativa dada su condición de proveedores de este tipo de contenidos en una plataforma de radio web. Por esta razón, se consideró de interés realizar una aproximación a las percepciones que los docentes presentan de las potencialidades educativas de una webradio; aproximación que complementa y enriquece la de los alumnos.

En busca de conocer las percepciones de los docentes en torno al potencial educativo de una webradio se tomó como referencia una muestra de conveniencia compuesta por un total de cinco individuos, todos ellos pertenecientes al área de Comunicação en el Departamento de Comunicação e Arte de la UA. Selección fundamentada en la hipótesis de que, dada su especialidad, dichos profesores presentarían un amplio conocimiento de los nuevos media, de sus posibilidades educativas, así como ofrecer una perspectiva crítica de los mismos.

En este sentido, para obtener la mayor información posible sobre la concepción que los profesores, como principales productores de contenidos educativos, tienen de las webradios se optó por la entrevista en profundidad como herramienta de investigación.

Una entrevista, de alrededor de 20 minutos de duración, que se estructuró en torno a tres bloques de preguntas: su percepción de la radio web como usuario eventual, su percepción de las posibilidades educativas de dicha plataforma y su percepción de las potencialidades que, el uso de dicha webradio, podría ofrecer para su docencia concreta.

Las aportaciones realizadas por los docentes durante el transcurso de dichas entrevistas fueron registradas en formato audio y revisadas. Esta revisión permitió extraer ideas para su aplicación en la siguiente fase de esta investigación (sobre una muestra representativa del colectivo de profesores), al tiempo que enriqueció el diseño de la programación para una futura webradio universitaria. 


\section{Resultados}

El trabajo desarrollado permitió profundizar en torno a las percepciones que, alumnos y profesores, tienen de los beneficios educativos de la puesta en marcha de una webradio en el ámbito de la comunidad universitaria de Aveiro. Unos resultados que fueron estructurados en dos bloques atendiendo a su divergencia muestral y metodológica: estudiantes y profesores.

\subsection{Estudiantes}

Los estudiantes encuestados se mostraron particularmente receptivos a la creación de una webradio en el contexto de la comunidad universitaria de Aveiro. En efecto, el $89 \%$ de los alumnos consideran de interés la existencia de dicha webradio y más de un $60 \%$ inclusive prevé la posibilidad de que dicha plataforma pueda convertirse en su emisora preferida.

A la hora de seleccionar el tipo de contenidos que les gustaría encontrar en esta plataforma académica de webradio, los estudiantes tienden a señalar aquellos más próximos al medio radiofónico convencional: contenidos de carácter informativo, musical y de divulgación científica (seleccionados por el $78 \%, 75 \%$ y $76 \%$ de los respondientes, respectivamente). 


\section{Gráfico 2. Preferencias de contenidos de los estudiantes para una webradio} universitaria.

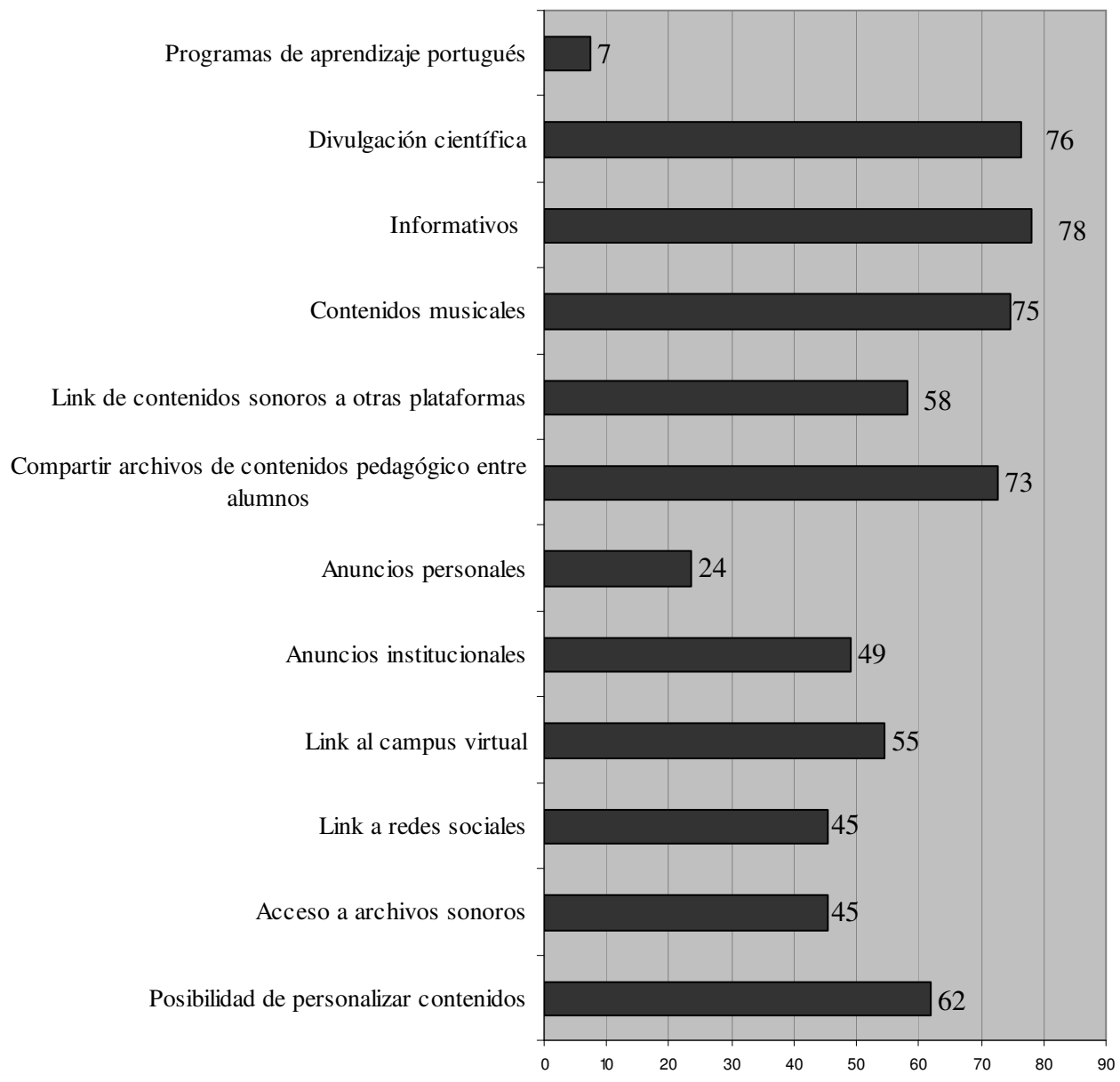

REDMARKA UIMA-Universidad de A Coruña - CIECID

Año III, Número 6, (2011), pp. 85-112

http://www.redmarka.org/

ISSN 1852-2300 
Fuente. Elaboración propia.

En lo que respecta a aquellos contenidos adaptados a las características de la radiofonía web, los estudiantes destacaron la inclusión de un espacio dedicado a compartir materiales de carácter docente (opción señalada por el $73 \%$ de los encuestados) o la vinculación de la webradio a otras plataformas que permitan enriquecer los contenidos con la incorporación del hipertexto, hiperaudio e hipermedia (opción seleccionada por el $58 \%$ de la muestra). Ambas preferencias, estrechamente relacionadas con la vertiente educativa de la radio, subrayan una proyección de los encuestados como prosumidores en el escenario de una webradio universitaria.

Entre los contenidos con menor preferencia por parte de los sujetos encuestados se encuentran la creación de un archivo sonoro o un repositorio de carácter académico online, especialmente si estos podcast se refieren al aprendizaje del portugués, o la inclusión de un enlace a las redes sociales (ambos señalados por un $45 \%$ de alumnos respectivamente). Precisamente, resulta destacable el reducido interés por las redes sociales en esta plataforma de webradio universitaria desde la perspectiva de la interacción y de la participación como principales ventajas del medio. Ventajas que se incrementan de atenderse a la relevante penetración del social network en los "nativos digitales".

Asimismo es preciso subrayar el hecho de que, pese a considerar las potencialidades educacionales de una radio universitaria en la red (consideración que está apoyada por el $87 \%$ de los individuos interrogados), las preferencias de los estudiantes de la muestra remiten a una utilización más próxima al entretenimiento que a un uso educativo propiamente dicho.

\subsection{Profesores}

Al igual que sucedía en el caso de los estudiantes, los profesores entrevistados consideraron de interés la puesta en marcha de una webradio universitaria en 
la comunidad académica de Aveiro. Interés que fue justificado con la necesidad de dotar de visibilidad a las actividades desarrolladas por la propia Universidad así como el tipo de trabajos realizados por sus investigadores. Una visibilidad de tipo interna, como un canal de apoyo a la difusión de actividades más allá del diario web de la universidad (de escasa lectura entre los alumnos), y externa, para hacer partícipe a una comunidad más amplia de los eventos que tienen lugar al abrigo de la institución académica.

A la hora de definir un tipo de contenidos o programación para esta webradio existe una total coincidencia entre los sujetos entrevistados al señalar en primer lugar la divulgación científica; una selección en consonancia con la necesidad de dotar de visibilidad a las actividades desarrolladas en el ámbito de la institución universitaria.

Otra tipología de contenidos que los entrevistados señalaron como relevante para esta webradio universitaria es la musical. La mayoría de los profesores se refirieron a dichos contenidos como base de la programación de esta plataforma de radio web universitaria, siempre y cuando su selección atendiese a un patrón no comercial que ofreciese la posibilidad de crear playlist alternativos a la radiofórmula. Inclusive, uno de los entrevistados señaló lo interesante de dicha plataforma para dar a conocer nuevos talentosque, inclusive, podrían pertenecer a la comunidad universitaria.

Ambos contenidos, divulgativos y musicales responden a una concepción bastante convencional de las radios universitarias, en la que la vertiente educativa queda relegada. De hecho, solamente uno de los entrevistados se refirió a la grabación de clases magistrales o determinados seminarios de interés para la comunidad académica, como contenidos adecuados para dicha plataforma.

No obstante, cuando se les interrogó directamente sobre la perspectiva didáctica de esta webradio, todos los profesores ratificaron sus potencialidades para la educación y la docencia. Entre éstas destacaron el desarrollo de contenidos como actividades extracurriculares de la asignatura como, por 
ejemplo, fomentar que los estudiantes produzcan piezas audiovisuales para la radio. Una posibilidad que entronca directamente con la idea de las radios universitarias como lugar para "criar um contexto semi profissional" (Entrevistado 3).

En este sentido, uno de los entrevistados se refirió al caso concreto del alumnado de la titulación en Novas Tecnologias da Comunicação y al interés de dotarlo de formación práctica a partir de la elaboración de contenidos para esta radio universitaria. Una práctica, de carácter curricular o extracurricular, que pondría en juego las competencias adquiridas en la disciplina de sonorización del segundo curso de la titulación.

Otra de las opciones señalada por los entrevistados fue la de la creación de un tipo de programas, que podrían denominarse magazines científicos, sobre un pequeño grupo de disciplinas con contenidos producidos a partir del resultado de la producción científica o de los trabajos académicos de su alumnado.

Un aprovechamiento educativo que entronca directamente con otra de las potencialidades educativas señaladas para esta webradio universitaria: que algunas materias/titulaciones, en particular las encaminadas al desarrollo de competencias de expresión oral y escrita o digitales, exijan los trabajos académicos en formato sonoro o audiovisual. Un cambio del papel al archivo de audio/vídeo que no sólo enriquecería el proceso de enseñanza-aprendizaje, sino que dotaría de contenidos a dicha plataforma de radio web.

Como se puede observar, los profesores entrevistados tienen presentes las potencialidades educativas de las radios universitarias aunque su asociación con las nuevas características del medio, adquiridas a partir de la radiomorfosis, todavía no resulta especialmente patente.

Prácticamente todas las ideas aportadas por los entrevistados podrían ponerse en marcha, aunque con menor facilidad de acceso y producción, en un medio analógico; circunstancia que refleja una cierta dificultad a la hora de concebir la radio en Internet como una plataforma multimedia. 
Al mismo tiempo, pese a ser conscientes de los beneficios que las webradios universitarias pueden aportar a la educación, particularmente desde la perspectiva de su proximidad a los estudiantes como oyentes-usuarios, "os alunos estão todo o dia com o computador, então existem mais possibilidades de que a usem [a webradio] e acedam aos seus conteúdos", les resulta complejo concretar dichos beneficios para su asignatura o asignaturas "Em disciplinas práticas [como la suya] não teria interesse".

Algunos entrevistados consideran posible su utilización en alguna de sus materias aunque responde a un uso divulgativo, para que la comunidad (universitaria o más amplia) conozca los trabajos-proyectos desarrollados, más que a una finalidad educativa en sentido estricto.

En definitiva, los profesores entrevistados consideran interesante la puesta en marcha de una webradio en el ámbito de la UA. Una webradio que conciben, fundamentalmente, como una plataforma de divulgación científica para una comunidad más amplia que la universitaria, en la que no deben faltar los contenidos musicales. Esta concepción deja relegada la vertiente educativa, objeto de estudio del presente trabajo.

\section{Conclusiones}

La aproximación efectuada en torno a las percepciones que docentes y discentes presentan de las potencialidades de una plataforma de radio web universitaria, en particular desde la perspectiva educativa, ha permitido extraer las siguientes conclusiones:

Estudiantes y profesores coinciden a la hora de considerar de interés la creación de una plataforma de radio web en el ámbito de la comunidad universitaria de Aveiro, e incluso una mayoría de alumnos (un 60\%) prevén la posibilidad de que se convierta en su emisora de referencia.

Una coincidencia que se repite a la hora de seleccionar los contenidos que les 
gustaría encontrar en dicha webradio. Así, docentes y discentes, se decantan por la divulgación científica y una programación de tipo musical como elementos base para una emisora web que opere en la UA.

Desde la perspectiva educativa propiamente dicha y, pese a no considerarla como prioritaria en su concepción de webradio, ambos colectivos subrayan algunas de sus potencialidades como configurar un espacio para compartir materiales pedagógicos entre alumnos o simular un contexto de práctica profesional. Una percepción de las potencialidades del medio que presenta algunas particularidades.

Todos los profesores destacaron el papel educativo de una webradio que opera en una comunidad universitaria, fundamentalmente, desde el punto de vista de la divulgación de trabajos académicos o el desarrollo de competencias relacionadas con la producción de contenidos. Papel que refleja una concepción de radio educativa todavía muy próxima a la del medio convencional.

Esta perspectiva en torno al papel educativo de la radio contrasta con su aplicación práctica a una materia concreta. En efecto, los docentes entrevistados consideran que, pese a sus potencialidades didácticas, la creación de una webradio en la UA apenas tendría proyección en su ejercicio docente.

Por su parte, pese a reconocer las potencialidades de la plataforma de radio web para implementar el proceso de enseñanza aprendizaje, e incluso subrayar aquellos contenidos que les reportaría un papel más activo, las preferencias de los estudiantes van encaminadas hacia una radio más próxima al entretenimiento que a un uso educativo propiamente dicho.

En definitiva, a pesar de las potencialidades que la radiofonía web ofrece para la educación, y su reconocimiento por parte de los principales públicos de una radio universitaria, todavía no existe una percepción completa de su aplicación práctica. Si bien ambos públicos, estudiantes y profesores, esbozan algunas de 
las utilidades didácticas de esta plataforma, éstas todavía resultan demasiado próximas al medio tradicional (desde el punto de vista sonoro) o al campus virtual (como plataforma de contenidos textuales e hipertextuales), obviando las capacidades de su nueva esencia multimedia. 


\section{Bibliografía}

Albarran, A.\& Pitts, G. (2001). Radio Broadcasting Industry. Boston. Allyn \& Bacon,

Baker, A. (2010). REVIEWING NET-ONLY COLLEGE RADIO: A CASE STUDY OF BROOKLYN COLLEGE RADIO, Journal of Radio \& Audio Media, 17 (1); pp. 109-125.

Corbett, J. (1993). RADIO DADA MANIFIESTO, Strauss, N. (ed.) Radio (text)e. Nueva York. Semiotexte; pp. 82- 92.

Costa Filho, I. (2009). CIBEROUVINTES DA UNIVERSIDADE FEDERAL DO CEARÁ: AS CONTRADIÇÕES DO USO SOCIAL DO RÁDIO PELA INTERNET, XI Congresso de Ciências da Comunicação na Região Nordeste. Intercom. Sociedade Brasileira de Estudos Interdisciplinares da Comunicação, [recuperado 23- 2-2011] de http://www.portcom.intercom.org.br/novosite/navegacaoDetalhe.php?opti on=trabalho\&id $=23518$

Desztich, R. \& Mcclung, S. (2007). INDIE TO AN EXTENT? WHY MUSIC GETS ADDED TO COLLEGE RADIO PLAYLIST, Journal of Radio \& Audio Media, 14 (2); pp. 196-211.

Faus, A. (1973). La Radio. Madrid. Guadiana,

Fidalgo Díez, D. (2009). LAS RADIOS UNIVERSITARIAS EN ESPAÑA. TRANSFORMACIÓN AL MUNDO DIGITAL, Telos, 80, [recuperado 3011-2010] de http://sociedadinformacion.fundacion.telefonica.com/telos/articulodocume nto.asp@idarticulo=2\&rev=80.htm

Fidler, R. (1997). Mediamorphosis: Understanding New Media. Thousand Oaks. Pine Forge Press.

González Conde, M.J. (2001). Información Universitaria en la Radio Pública: Radio 5-Todo Noticias. Disertación doctoral, Universidad Complutense de Madrid, España, [recuperado 17-2-2011] de http://eprints.ucm.es/tesis/19972000/S/3/S3030301.pdf

Islas-Carmona, O. (2008). EL PROSUMIDOR. EL ACTOR COMUNICATIVO DE LA SOCIEDAD DE LA UBICUIDAD, Palabra Clave, 11 (1) , [recuperado 
11-3-2011]

de

http://revistas.unisabana.edu.co/index.php/palabraclave/article/viewArticl

e/1413/172.

Kuhn, F. (2001). O RÁDIO NA INTERNET: RUMO À QUARTA MÍDIA, XXVII Congresso Brasileiro de Ciências da Comunicaçã, Campo Grande, [recuperado

http://revistas.unisabana.edu.co/index.php/palabraclave/article/viewArticl e/1413/172

Mcclung, S., Pompper, D. \& Kinnally, W. (2007). THE FUNCTIONS OF RADIO FOR TEENS: WHER(E RADIO FITS AMONG YOUTH MEDIA CHOICES, Atlantic Journal of comunication, 15 (2); pp. $103-119$.

Nyre, L. \& Ala-Fossi, M. (2008). THE NEXT GENERATION PLATFORM: COMPARING AUDIENCE REGISTRATION AND PARTICIPATION IN DIGITAL SOUND MEDIA, Journal of Radio \& Audio Media, 15 (1); pp. 41-58.

Perona Páez, J. (2009). EDU-WEBS RADIOFÓNICAS: EXPERIENCIAS ESPAÑOLAS DE EDUCACIÓN EN MEDIOS, Comunicar, 17(33); pp. 107-114.

Pessoa, S. (2008). WEBRADIO AMIRT: A EXPERIÊNCIA DE ESTUDANTES NA TRANSMISSÃO RADIOFÔNICA ON-LINE, Revista de Comunicação, 9 [recuperado 20-2-2011] de http://www2.pucpr.br/reol/index.php/COMUNICACAO?dd1=1975\&dd99= view

Prata, N. (2008). Webradio: Novos Géneros, Novas Formas de Interacção. Disertación doctoral, Universidade Federal de Minas Gerais, Brasil [recuperado 12-2-2011]

de http://www.bibliotecadigital.ufmg.br/dspace/bitstream/1843/AIRR7DDJD8/ 1/nair_prata_tese.pdf

Prensky, M (2001). DIGITAL NATIVES, DIGITAL IMMIGRANTS PART 1, On the Horizont, 9 (5); pp. 1-6.

Priestman, C. (2002). Web Radio - Radio Prodution for Internet Streaming. Oxford. Focal Press.

Rose, B. \& Lenski, J. (2007). THE INFINITE DIAL 2007: RADIO'S DIGITAL 
PLATFORMS, ONLINE, SATELLITE, HD RADIO AND PODCASTING, [recuperado 26-2-2011]

de http://repositorium.sdum.uminho.pt/bitstream/1822/6251/1/pedro\%20port ela.pdf

Rothenbuhler, E.W. (1981). COMMERCIAL RADIO AND POPULAR MUSIC: PROCESS OF SELECTION AND FACTORS OF INFLUENCE, LIUI, J. (ed.) Popular Music and Communication. Newbury Parl. Sage; pp. 79-95.

Sauls, S. (1995). COLLEGE RADIO, Annual Joint Meetings of the Popular Culture Association. Philadelphia. American Culture Association.

Sauls, S. (2000). The culture of American college radio. Ames. lowa State University.

Sauls, S. (1998). THE ROLE OF ALTERNATIVE PROGRAMMING IN COLLEGE RADIO, Studies in Popular Cultura, 20 (1).

Teixeira, M. \& Da Silva, B. (2009a). EXPERIÊNCIAS DO RÁDIO NA EDUCAÇÃO ONLINE, Silva, B., Almeida, L. , Lozano, A. \& Uzquiano, M. (orgs) Actas do $X$ Congresso Internacional Galego- Português de Psicopedagogia. Braga. Edições CIED; pp. 5726-5736.

Teixeira, M. \& Da Silva, B. (2009). RÁDIO WEB: EDUCAÇÃO, COMUNICAÇÃO E CIBERCULTURA NO UNIVERSO ACADÉMICO PORTUGUÊS, Dias, P. \& Osório, A. J. (org.) Challenges 2009 : actas da Conferência Internacional de TIC na Educação. Braga. Universidade; pp. 193-202.

Teixeira, M., Perona Páez, J.J.\& Daher, M. (2010). A RÁDIO WEB UNIVERSITÁRIA COMO MODALIDADE EDUCATIVA AUDIOVISUAL EM CONTEXTO DIGITAL. OS CASOS DA ESPANHA E DE PORTUGAL, Pretto, N. \& Tosta, S. (orgs.) Do MEB à WEB. O Rádio na Educação. Belo Horizonte. Autêntica; pp. 175-194.

Machado Velho, P. (s/d) A LINGUAGEM DO RÁDIO MULTIMÍDIA, [recuperado 21-3-2011] de http://www.bocc.ubi.pt/pag/bocc-velho-linguagem.pdf

Waits, J.C. (2008). DOES 'INDIE' MEAN INDEPENDENCE? FREEDOM AND RESTRAINT IN A LATE 1990S US COLLEGE RADIO COMMUNITY, The Radio Journal. International Studies in Broadcast and Audio Media, 5 (23); pp. 83-96.

Wall, B. (2007). FINDING AN ALTERNATIVE: MUSIC PROGRAMMING IN US 
COLLEGE RADIO, The Radio Journal. International Studies in Broadcast and Audio Media, 5 (1); pp. 35-54.

Wallace, D. (2008). REINVENTING THE WHEEL VS. GRINDING THE SAME OLD AXE: AN ETHNOGRAPHIC VIEW OF THE STUDENTS AND COMMUNITY MEMBERS AT A MASSACHUSETTS COLLEGE RADIO STATION, Westminster Papers in Communication and Culture, 5 (1); pp. 1744-6716.

Para citar este artículo

Piñeiro-Otero, Teresa - Ramos, Fernando (29-07-2011). POTENCIALIDADES EDUCATIVAS DE LAS WEBRADIOS UNIVERSITARIAS. UNA APROXIMACIÓN DESDE LA PERSPECTIVA DE DOCENTES Y DISCENTES.

REDMARKA - CIECID - Unidad de Investigación en Marketing Aplicado-Universidad de A Coruña

Año III, Número 6, V1, pp.85-112

ISSN 1852-2300

URL del Documento: cienciared.com.ar/ra/doc.php?n=1492

URL de la Revista: cienciared.com.ar/ra/revista.php?wid=39 